\title{
Anemia among HIV Infected Children on Highly Active Anti Retroviral Therapy in Wolaita Zone, South Ethiopia: a facility based cross sectional study
}

\author{
Mesay Lema Geleta \\ Wolaita Sodo University \\ Fithamlak Bisetegn Solomon \\ Wolaita Sodo University \\ Efrata Girma Tufa \\ Wolaita Sodo University \\ Fekadu Elias Sadamo \\ Wolaita Sodo University \\ Samson Kastro ( $\square$ tasamsona@gmail.com ) \\ Wolaita Sodo University https://orcid.org/0000-0002-7687-4674
}

Research article

Keywords: Anemia, Anti retroviral therapy, HIV, Children, Wolaita, Ethiopia

Posted Date: October 1st, 2019

DOI: https://doi.org/10.21203/rs.2.15468/v1

License: (c) (i) This work is licensed under a Creative Commons Attribution 4.0 International License.

Read Full License 


\section{Abstract}

Background Anemia is a global public health problem and most of the HIV positive people become anemic at some point in the course of the disease. We lack adequate evidence on the magnitude of anemia among children on highly active anti retroviral therapy in Ethiopia and particularly in South Ethiopia. Thus, this study aimed at determining the proportion and associated factors of anemia among children on highly active anti retroviral therapy in Wolaita zone, South Ethiopia.

Method a facility based cross-sectional study has been conducted from November to December 2018 on 256 children 6 months to 14 years of age who were on Anti retroviral therapy. Data were collected through an interview with caregivers and review of medical records. CD4+ cell count was analyzed using FACS caliber and Hemoglobin level was measured with Hem cue 301 analyzer. Stool sample was examined for presence of intestinal parasites by direct wet mount technique. Data analyzed with Stata version 14.0 were conveyed in mean and standard deviation of the mean, median and inter-quartile range. Multivariate analysis was carried out to identify independent predictors of the outcome variable. Adjusted Odds Ratio with $95 \%$ confidence interval was reported.

Results The magnitude of anemia was found to be $38.8 \%$. Taking Co-trimoxazole antibiotic prophylaxis $(A O R=.45 ; 95 \% \mathrm{Cl}: .21, .95)$, caregivers' not receiving nutritional counseling (AOR=09; 95\% Cl: .01, .98) and presence of intestinal parasites $(\mathrm{AOR}=3.10 ; 95 \% \mathrm{Cl}: 1.39,6.88)$ were associated with anemia.

Conclusion The magnitude of anemia obtained in this study is a moderate public health problem. Health education programs in Anti Retroviral Therapy clinics should be targeted at appropriate dietary practice, and appropriate hand washing and other hygienic practices to prevent intestinal parasitic infections. Cotrimoxazole prophylaxis should give given to all eligible children based on the recommendation.

\section{Background}

Anemia is a condition in which the number of red blood cells is insufficient to meet the body's physiologic needs and consequently diminish the oxygen-carrying capacity. In addition to iron deficiency, other nutritional deficiencies, acute and chronic inflammations, parasitic infections, and inherited or acquired disorders that affect hemoglobin synthesis, red blood cell production or survival can cause anemia [1].

Children with Human Immunodeficiency Virus (HIV) are exposed to macro and micro nutrient deficiencies; anemia being the commonest [2,3]. Anemia is a critical health concern affecting growth and energy levels adversely, and it highly damages the immune mechanisms $[4,5]$.

Anemia affects both developing and developed countries and thus it is a global public health problem [6]. Globally, roughly 1.3 to 2.2 billion people suffer from anemia and in developing countries $50 \%$ of women and children are anemic [7]. An estimated $63 \%-95 \%$ of people living with HIV become anemic at some point in the course of the disease [8]. In India the prevalence of anemia among HIV infected children was reported to be $47.1 \%$ [9]. A prevalence level of $69.1 \%$ was reported among HIV infected children in 
Democratic Republic of Congo [10]. In Ethiopia the prevalence of anemia among HIV infected children ranges between $16.2 \%$ and $39.1 \%[11,12,13]$. A combination of environmental and host factors predispose HIV infected children to anemia.

We have very limited evidence on the prevalence of anemia among children on Highly Active Anti Retroviral Therapy (HAART) in Ethiopia and there is a wide variation in the reported prevalence. Thus the main aim of this study was to estimate the proportion of anemia among HIV infected children on HAART and to identify factors associated with the outcome in Anti Retroviral Therapy (ART) clinics of Wolaita zone, South Ethiopia.

\section{Methods}

\section{Study Setting}

A facility based cross-sectional study was conducted from November to December 2018 at ART Clinics of Wolaita zone. There are 16 health facilities, 12 health centers and 4 hospitals, which provide ART service. Wolaita zone has a latitude and longitude of $6^{0} 54^{\prime} \mathrm{N} 37^{0} 45^{\prime} \mathrm{E}$ with an average elevation of 1850 meters above sea level.

\section{Population and sampling}

Children, 6 months to 14 years of age, on HAART paired with their mothers/caretakers who lived in the study area for at least half a year were included in this study. Since there are 262 children on HAART attending ART clinics of Wolaita zone, sample size was not calculated and all eligible children were included. Children who were on anemia treatment were excluded from the study.

\section{Data Collection}

A structured and interviewer administered questionnaire was used to collect data. Medical records were reviewed and presence of opportunistic infections, baseline CD4+ count and World Health Organization (WHO) clinical stage were recorded to complement the primary data. Twenty four hours recall was used to assess dietary diversity. Nurses and laboratory technicians were recruited for interview and laboratory analysis respectively. BSc nurses were recruited for supervision. Two days training was given for data collectors on the art of interviewing. Trained laboratory technicians were employed and additional training was given. The questionnaire was pretested on $5 \%$ of the sample size at Shone Primary Hospital which is not part of the actual data collection. Close supervision was under taken during data collection in a way avoiding bias. Standardized operating procedures were strictly followed during blood sample collection, storage and analytical process. 


\section{Laboratory analysis}

Three $\mathrm{ml}$ of venous blood sample was collected using EDTA anti-coagulated test tube for CD4+ cell count and hemoglobin analyses. CD4+ cell count was analyzed using FACS caliber and Hemoglobin level was measured with Hem cue 301 analyzer, Angelholm, Sweden. Stool sample was taken and examined by direct wet mount technique to detect present of intestinal parasites microscopically.

\section{Operational Definitions}

Anemia: is established if the hemoglobin level is below $11 \mathrm{~g} / \mathrm{dl}$ for $6-59$ months, $<11.5 \mathrm{~g} / \mathrm{dl}$ for $5-$ 11 years and $<12 \mathrm{~g} / \mathrm{dl}$ for $12-14$ years and adjusted for altitude [19].

\section{Statistical analysis}

Data were entered into Epi data version 3.1 and exported to Stata version 14.0 for analyses. The data were conveyed in mean and standard deviation of the mean, median and inter-quartile range. Normality was checked for all continuous variables. Hosmer-Lemeshow good-of-fit test and Receiver Operating Characteristics (ROC) test were carried out to have confidence in the regression model. Collinearity was diagnosed, and there was no multicollinearity among the exposure variables included in the model. We used logistic regression analysis to identify exposure variables with association to the outcome variable. Exposures and covariates with $p<0.25$ during the bivariate analysis were included in multivariate analysis to increase the power of the model. Multivariate analysis was carried out to control for potential confounders and identify independent predictors of the outcome variable. Adjusted Odds Ratio (AOR) with $95 \%$ confidence interval $(\mathrm{Cl})$ was reported. The level of significance was set at $p<0.05$.

\section{Results}

\section{Socio-Demographic Characteristics}

Overall, 256 children On HAART participated in the study with a response rate of $97.7 \%$. More than half, $140(54.7 \%)$ were girls. The median age was $12(10-14)$ years, and $144(56.2 \%)$ of them were between 12-14 years of age. About three in four, $192(75.0 \%)$ were rural dwellers. One-third, $85(33.2 \%)$ of the caregivers did not attend formal education and 74 (28.9\%) were employed. About two third, 171 (66.8\%) of the households use either pipe or public tap water for drinking (Table 1).

\section{Clinical and Immunological Characteristics}

One hundred seventy one (66.8\%) were on HAART for more than 60 months. A lithe higher than half, 132 (51.6 \%) were in WHO stage 3 at baseline and nearly all, 251 (98.0\%) were in WHO stage T1. Forty two 
(16.4\%) switched their drug regimen and 44 (17.2\%) had some form of opportunistic infection. 73 (28.5

$\%)$ were taking co-trimoxazole prophylaxis. The median CD4+ count was $746.5(516-1007) \mathrm{cells} / \mathrm{mm}^{3}$ currently, and majority of them, $182\left(85.8 \%\right.$ ) had a CD4+ count of greater than 350 cells $/ \mathrm{mm}^{3}$ (Table 2).

\section{Nutritional, Hygienic and Parasitological Characteristics}

The majority of caregivers, 210 (82\%) affirmed getting dietary advice. Nearly two third, $167(65.2 \%)$ of the children often eat less than three meals a day and $115(44.9 \%)$ had dietary diversity of fewer than four. Most, 210 (82.0\%) of the children had no intestinal parasitic infection (Table 3).

\section{Proportion and factors Associated with Anemia}

The mean \pm (standard deviation) hemoglobin level of the study participants was $12.2 \pm$ (1.4). The proportion of anemia was $38.8 \%$. Children taking co-trimoxazole antibiotic prophylaxis had $55 \%$ lower chance of being anemic than those who were not taking Co-trimoxazole (AOR $=.45 ; 95 \% \mathrm{Cl}: 21, .95)$. Children whose caregivers were given nutritional counseling had $91 \%$ lesser chance of having anemia (AOR $=09 ; 95 \% \mathrm{Cl}: .01, .98)$. The odd of being anemic was 3 times higher for children who had intestinal parasitic infection than their counterparts ( $\mathrm{AOR}=3.10 ; 95 \% \mathrm{Cl}: 1.39,6.88)$.

\section{Discussion}

In this study, the prevalence of anemia among children on HAART was found to be $38.8 \%$. The prevalence of anemia in this study is a moderate public health problem according to the WHO classification of anemia [1]. This finding is comparable with a similar study conducted in different parts of Ethiopia (35\%) [14] and 39.1\% [15]. However, it is lower compared to a study conducted in southwest Ethiopia (53.1\%) [16]. Lower prevalence was reported in different parts of Ethiopia (16.2\%) [11], (10\%) [17] and (22.2\%) [12]. This divergence might be due to variation in the culture, dietary practice and socio-economic status across different segments of the population within the country.

Children who were taking Co-trimoxazole antibiotic prophylaxis had a lesser odds of being anemic compared to those who did not. This is in agreement with studies from Ethiopia and Gambia [11, 18]. One of the most important mechanisms of anemia in HIV infection is failure of erythropoiesis. Co-trimoxazole may probably reduce the levels of cytokines that impair erythropoiesis, by reducing immune activation as well as by preventing infections [19].

Children whose caregivers did not receive nutritional counseling were more likely to be anemic compared to their counter parts. This finding is in agreement with a study conducted in Hawassa referral hospital where children whose caregivers did not receive nutritional counseling had higher odds of being anemic [20]. Better information on the key dietary recommendations might have improved the caregiver's practice of child feeding. 
In consistent with studies from Ethiopia [12] and Tanzania [21], children with intestinal parasitic infections had much higher odds to have anemia compared with those children who were not infected with intestinal parasitic infections. This might possibly be due to the attachment of the worm in the intestine to the intestinal mucosa causing intestinal necrosis and blood loss. Chronic infections may also lead to anemia which results from excessive iron loss [4].

Unlike many other studies, this study did not found an association between lower CD4+ cell count and anemia. This finding disagrees with other similar study from Addis Ababa (Central Ethiopia) [12]. This difference might be due to the less number of children with lower CD4+ count involved in this study.

\section{Study limitations}

Regarding dietary habit, certain level of recall bias was expected; data collectors aware of the cultural issues collected the data to reduce recall bias. Data on the duration of HAART, baseline CD4+ count, WHO stage, drug regimen, taking Co-trimoxazole and presence of opportunistic infections were taken from the patients charts. Since the health facilities record patient's information consistently and accurately, we believe that the data is unbiased. CD4+ count was not done for 44 children due to lack of reagent. Since the reagent comes as a donation to the country, we couldn't access it at a market.

\section{Conclusions}

The magnitude of anemia among children on HAART in the study area is a moderate public health problem. Anemia was significantly associated with caregivers' not receiving nutritional counseling and intestinal parasitic infections. Taking Co-trimoxazole antibiotic prophylaxis was protective against anemia. Health education programs in ART clinics should be given with due focus on appropriate dietary practice, appropriate hand washing practice and other hygienic practices to prevent intestinal parasitic infections. Co-trimoxazole prophylaxis should give given to all eligible children based on the recommendation.

\section{Abbreviations}

ABC: Abacavir, AIDS: Acquired Immune Deficiency Syndrome, AOR: Adjusted Odds Ratio, ART: Anti Retroviral Therapy, AZT: Zidovudine, COR: Crude Odds Ratio, ETB: Ethiopian Birr, HAART: Highly Active Anti Retroviral Therapy, HIV: Human Immunodeficiency Virus, OI: Opportunistic Infections, ROC: Receiver Operating Characteristics, TDF: Tenofovir Disoproxil Fumarate, WHO: World Health Organization

\section{Declarations}

\section{Ethics approval and consent to participate}


Ethical clearance for the study was obtained from the Ethical review committee at College of Health Science and Medicine in Wolaita Sodo University in Ethiopia. The ethical clearance letter was written to Wolaita zone health department which is the highest government body in the area for health and health research conducts. Permission was granted from Wolaita zone health department and its lower health administrative structures to conduct the study. Finally, informed written consent was taken from caregivers since we can't obtain consent from children under 18 years of age. The data was kept anonymous.

Consent for publication-Not applicable

\section{Availability of data and materials}

The dataset analyzed for this study is available from the corresponding author on reasonable request.

\section{Competing interests}

The authors declare that they have no competing interests.

Funding: This study was financially supported byWolaita Sodo University through graduate level research program. Neither of the parties had role in the design, conduct and decision to publish this research work.

\section{Authors' contributions}

ML conceived the study, designed the protocol, coordinated data collection, analyzed the data interpreted the findings and reviewed the manuscript. SK contributed in the design, analysis, interpretation of the findings, and drafted the manuscript. FS, EG and FE contributed in design, analysis, interpretation of the findings, and reviewed the progressive draft manuscript. All authors read and approved the final version of the manuscript.

\section{Authors' information}

$M L$ is a human nutritionist by training. She studied MSc in human nutrition and currently working as a service quality officer in the College of Health Sciences and Medicine at Wolaita Sodo University in Ethiopia. SK is a human nutritionist by training. He is lecturer at School of Public Health at WSU. FG is a medical microbiologist by training. He is an assistant professor of medical microbiology in the Department of Medical Laboratory at WSU. EG is a human nutritionist by training. She is lecturer at School of Public Health at WSU. FE is a public health nutritionist by training. He is lecturer at School of Public Health at WSU. 


\section{Acknowledgements}

We are grateful to Wolaita Sodo University for financially supporting the study. We would like to thank School of Public Health at Wolaita Sodo University for facilitating the research programme. We acknowledge Wolaita zone health department and its lower administrative structures for official permission. We are grateful to the caregivers and children for their consent to participate in the study. We also thank our responsible data collectors.

\section{References}

1. WHO, Haemoglobin concentrations for the diagnosis of anaemia and assessment of severity. Vitamin and Mineral Nutrition Information System. Geneva, World Health Organization, 2011 (WHO/NMH/NHD/MNM/11.1) (http://www.who.int/vmnis/indicators/haemoglobin.pdf, accessed 15/01/2019).

2. Sireesha, G. and N. Rajani, Nutritional status of Girls through Anthropometric and Dietary Assessment. IOSR J of Dental and Medical Sciences, 2015. 14(11): p. 73-78.

3. Prendergast, A. J. and J. H. Humphrey, The stunting syndrome in developing countries. Paediatrics and international child health, 2014. 34(4): p. 250-265.

4. WHO, Iron deficiency anemia. assessment, prevention, and control. A guide for programme managers. Geneva, World Health Organization, 2001: p. 47-62.

5. WHO, The world health report 2002: reducing risks, promoting healthy life. Geneva, World Health Organization, 2002.

6. Haas, J. D. and T. Brownlie IV, Iron deficiency and reduced work capacity: a critical review of the research to determine a causal relationship. The Journal of nutrition, 2001. 131(2): p. 676S-690S.

7. Stevens, G. A., et al., Global, regional, and national trends in haemoglobin concentration and prevalence of total and severe anaemia in children and pregnant and non-pregnant women for 1995-2011: a systematic analysis of population-representative data. The Lancet Global Health, 2013. 1(1): p. e16-e25.

8. Mehta, S., S. Jutur, and D. Gautam, Hematologic Manifestations of HIV/AIDS. Medicine Update, 2011. 9: p. 484-90.

9. Shet A., et al., Anemia, diet and therapeutic iron among children living with HIV: a prospective cohort study. BMC Pediatr, 2015; 15: 164.

10. Mwadianvita C.K, et al., Study of anemia in HIV positive children naïve to antiretroviral treatment in Lubumbashi, Democratic Republic of Congo. Pan Afr Med J, 2014; 17(46): doi: 10.11604/pamj.2014.17.46.2046.

11. Enawgaw, B., et al., Prevalence and associated risk factors of anemia among HIV infected children attending Gondar university hospital, Northwest Ethiopia: a cross sectional study. BMC hematology, 2015. 15(1): p. 12. 
12. Mihiretie, H., B. Taye, and A. Tsegaye, Magnitude of anemia and associated factors among pediatric HIV/AIDS patients attending Zewditu Memorial Hospital ART Clinic, Addis Ababa, Ethiopia. Anemia, 2015.

13. Teklemariam, Z., H. Mitiku, and F. Mesfin, Prevalence of anemia and nutritional status among HIVpositive children receiving antiretroviral therapy in Harar, eastern Ethiopa. HIV/AIDS (Auckland, NZ), 2015. 7: p. 191

14. Ferede, G. and Y. Wondimeneh, Prevalence and related factors of anemia in HAART-naive HIV positive patients at Gondar University Hospital, Northwest Ethiopia. BMC Blood Disorders, 2013. 13(1): p. 8

15. Zelalem T, H. M., Firehiwot M, Prevalence of anemia and nutritional status among HIV-positive children receiving antiretroviral therapy in Harar, eastern Ethiopa. HIV/AIDS-Research and Palliative Care, 2015. 7: p. 191-196

16. Abebe, M. and F. Alemseged, Hematologic abormalities among children on Haart, in Jimma university specialized hospital, southwestern ethiopia. Ethiopian Journal of Health Sciences, 2009. 19(2)

17. Hylemariam Mihiretie Mengist1, Bineyam Taye2, Aster Tsegaye2, Intestinal Parasitosis in Relation to CD4+T Cells Levels and Anemia among HAART Initiated and HAART Naive Pediatric HIV Patients in a Model ART Center in Addis Ababa, Ethiopia. PLOS ONE, 2015. 10.1371: p. 1-14

18. Prendergast A., et al., Improved growth and anemia in HIV-infeted children taking cotrimoxazole prophylaxis. Clin Infec Dis, 2011; 52(7): 953-7

19. Kreuzer KA, et al., Inadequate erythropoietin response to anemia in HIV patients: relationship to serum levels of tumor necrosis factor-alpha, interleukin -6 and their soluble receptors. Br J Haematol, 1997; 96:235-9

20. Wondimu, W. B., Nutritional Status and Associated Factors in Human Immunodeficiency Virus Infected Children in Hawassa University Referral Hospital Hawassa City, SNNPR,Ethiopia. 2nd National Nutrition Research Dissemination Conference, 2014

21. Makubi, A. N., et al., Risk factors for anaemia among HIV infected children attending care and treatment clinic at Muhimbili National Hospital in Dar es Salaam, Tanzania. Tanzania Journal of Health Research, 2012; 14(1)

\section{Tables}

Table 1 Socio-demographic characteristics of children and caregivers involved in this study at Wolaita zone in South Ethiopia, December 2018 


\begin{tabular}{|c|c|c|c|}
\hline Variables $(n=256)$ & & Frequency & Percent \\
\hline \multirow[t]{2}{*}{ Sex } & Male & 116 & 45.3 \\
\hline & Female & 140 & 54.7 \\
\hline \multirow[t]{2}{*}{ Age (months) } & 6 month to 11 years & 112 & 43.75 \\
\hline & $12-14$ years & 144 & 56.2 \\
\hline \multirow[t]{2}{*}{ Residence } & Rural & 192 & 75.0 \\
\hline & Urban & 64 & 25.0 \\
\hline \multirow[t]{4}{*}{ Educational status of care giver } & No formal education & 85 & 33.2 \\
\hline & Primary (1-8) & 87 & 34.0 \\
\hline & Secondary (9-12) & 23 & 9.0 \\
\hline & Above secondary & 61 & 23.8 \\
\hline \multirow[t]{5}{*}{ Occupation of caregiver } & Unemployed & 46 & 18.0 \\
\hline & Government /NGO employee & 74 & 28.9 \\
\hline & Merchant & 60 & 23.4 \\
\hline & Daily laborer & 44 & 17.2 \\
\hline & Farmer & 32 & 12.5 \\
\hline \multirow[t]{3}{*}{ Monthly income of household } & $\leq 2000$ ETB & 138 & 53.9 \\
\hline & 2001-4000 ETB & 73 & 28.5 \\
\hline & $>4000$ ETB & 45 & 17.6 \\
\hline \multirow[t]{3}{*}{ Source of drinking water } & Pipe/tap & 171 & 66.8 \\
\hline & Protected well/spring & 69 & 27.0 \\
\hline & Unprotected well/spring/river & 16 & 12.9 \\
\hline \multirow[t]{3}{*}{ Ways of treating water } & Boiling & 31 & 12.1 \\
\hline & Using chemicals & 192 & 75.0 \\
\hline & Filtration & 33 & 12.9 \\
\hline
\end{tabular}

Table 2 Clinical and immunological characteristics of children involved in this study at Wolaita zone in South Ethiopia, December 2018 


\begin{tabular}{llcc}
\hline Variables ( $\mathbf{n = 2 5 6 )}$ & & Frequency & Percent \\
\hline Duration of HAART & $<60$ months & 85 & 33.2 \\
& $\geq 60$ months & 171 & 66.8 \\
Baseline WHO stage & Stage 1 & 51 & 19.9 \\
& Stage 2 & 49 & 19.1 \\
& Stage 3 & 132 & 51.6 \\
& Stage 4 & 24 & 9.4 \\
Current WHO stage & T1 b & 251 & 98.0 \\
& T2/T3 c & 5 & 2.0 \\
Current drug regimen & TDF based & 18 & 7.0 \\
& AZT based & 225 & 87.9 \\
Opportunistic infections & ABC based & 13 & 5.1 \\
& Yes & 44 & 17.2 \\
Taking Co-trimoxazole & No & 212 & 82.8 \\
& Yes & 73 & 28.5 \\
Baseline CD4 count (n=240) & No & 183 & 71.5 \\
& S200 & 31 & 12.9 \\
& $201-350$ & 34 & 14.2 \\
Current CD4 count $(\mathrm{n}=212)$ & $>350$ & 175 & 72.9 \\
& $\leq 200$ & 9 & 4.3 \\
& $201-350$ & 21 & 9.9 \\
& $>350$ & 182 & 85.8 \\
\hline
\end{tabular}

b on treatment stage $1,{ }^{c}$ on treatment stage $2 / 3$

Table 3 Dietary, hygienic and parasitological characteristics of children and caregivers involved in this study at Wolaita zone in South Ethiopia, December 2018 


\begin{tabular}{llcc}
\hline Variables $(\mathbf{n}=\mathbf{2 5 6})$ & & Frequency & Percent \\
\hline Dietary counseling for care giver & Yes & 252 & 98.4 \\
& No & 4 & 1.6 \\
Receiving nutritional support & Yes & 143 & 55.9 \\
& No & 113 & 44.1 \\
Number of meals per day & Sthree & 129 & 50.4 \\
& >three & 127 & 49.6 \\
Skip regular meals & Yes & 127 & 49.9 \\
\multirow{4}{*}{ Dietary diversity } & No & 129 & 50.4 \\
& $<4$ & 115 & 44.9 \\
Intestinal parasitic infection & $\square 4$ & 141 & 55.1 \\
& Yes & 45 & 17.6 \\
& No & 211 & 82.4 \\
\hline
\end{tabular}

Table 4 Factors associated with anemia among children involved in this study at Wolaita zone in South Ethiopia, December 2018 


\begin{tabular}{|c|c|c|c|c|}
\hline Variable $(n=256)$ & Anemic & Non-anemic & $\operatorname{COR}(95 \% \mathrm{Cl})$ & AOR (95\% Cl) \\
\hline \multicolumn{5}{|l|}{ Age } \\
\hline 6 month to 11 years & $37(33.04 \%)$ & $75(66.96 \%)$ & $.67(.40,1.12)$ & $1.08(.56,2.12)$ \\
\hline $12-14$ years & $61(42.36 \%)$ & $83(57.64 \%)$ & 1 & 1 \\
\hline \multicolumn{5}{|l|}{ Occupation } \\
\hline Unemployed & 14(30.43) & $32(69.57 \%)$ & $.35(.16, .77)$ & $.31(.09,1.10)$ \\
\hline Employed & $41(55.41 \%)$ & $33(44.59 \%)$ & 1 & 1 \\
\hline Merchant & $17(28.33 \%)$ & $43(71.73 \%)$ & $.31(.15, .66)$ & $.63(.21,1.86)$ \\
\hline Daily laborer & $12(27.27 \%)$ & $32(72.73 \%)$ & $.30(.13, .68)$ & $.45(.14,1.49)$ \\
\hline Farmer & $14(43.75 \%)$ & $18(56.25 \%)$ & $.62(.27,1.44)$ & $1.05(.26,4.29)$ \\
\hline \multicolumn{5}{|l|}{ Education } \\
\hline No formal education & $33(38.82 \%)$ & $52(61.18 \%)$ & 1 & \\
\hline Primary $(1-8)$ & $26(29.89 \%)$ & $61(70.11 \%)$ & $.67(.36,1.27)$ & $.60(.25,1.40)$ \\
\hline Secondary $(9-12)$ & $4(17.39 \%)$ & $19(82.61 \%)$ & $.33(.10,1.06)$ & $.27(.06,1.18)$ \\
\hline Above secondary & $35(57.38 \%)$ & $26(42.62 \%)$ & $2.12(1.08,4.14)$ & $.98(.29,3.29)$ \\
\hline \multicolumn{5}{|l|}{ Water treatment } \\
\hline Boiling & $10(32.26 \%)$ & $21(67.74 \%)$ & $.68(.30,1.53)$ & $.66(.24,1.82)$ \\
\hline Chemicals & $79(41.15 \%)$ & $113(58.85 \%)$ & 1 & 1 \\
\hline Filtration & $9(27.27 \%)$ & $24(72.73 \%)$ & $.54(.24,1.22)$ & $.43(.16,1.18)$ \\
\hline \multicolumn{5}{|l|}{ Duration of HAART } \\
\hline$<60$ months & $27(31.76 \%)$ & $58(68.24 \%)$ & $.66(.38,1.13)$ & $.92(.46,1.83)$ \\
\hline$\geq 60$ months & $71(41.52 \%)$ & $100(58.48 \%)$ & 1 & \\
\hline \multicolumn{5}{|l|}{ Baseline WHO stage } \\
\hline Stage 1 & $13(25.49 \%)$ & $38(74.51 \%)$ & $.60(.29,1.23)$ & $.63(.26,1.49)$ \\
\hline Stage 2 & $22(44.90 \%)$ & $27(5 !$ & $1.43(.73,2.77)$ & $1.49(.64,3.44)$ \\
\hline Stage 3 & $48(36.36 \%)$ & $84(63.64 \%)$ & 1 & \\
\hline Stage 4 & $15(62.50 \%)$ & $9(37.50 \%)$ & $2.92(1.19,7.17)$ & $2.15(.73,6.30)$ \\
\hline \multicolumn{5}{|c|}{ Opportunistic infections } \\
\hline Yes & $14(56.00 \%)$ & $11(44.00 \%)$ & $2.23(.97,5.13)$ & $2.47(.88,6.97)$ \\
\hline No & $84(36.36 \%)$ & $147(63.64 \%)$ & 1 & \\
\hline \multicolumn{5}{|c|}{ Taking Co-trimoxazole } \\
\hline Yes & $22(38.28 \%)$ & $51(69.86 \%)$ & $.61(.34,1.08)$ & $.45(.21, .95)^{\star}$ \\
\hline No & $76(41.53 \%)$ & $107(58.47 \%)$ & 1 & \\
\hline \multicolumn{5}{|c|}{ Nutritional counseling } \\
\hline Yes & $95(37.70 \%)$ & $157(62.30 \%)$ & $.20(.02,1.97)$ & $.09(.01, .98)^{\star}$ \\
\hline No & $3(75.00 \%)$ & $1(25.00 \%)$ & 1 & \\
\hline \multicolumn{5}{|l|}{ Dietary diversity } \\
\hline$<4$ & $39(34.21 \%)$ & $75(65.79 \%)$ & $.73(.44,1.22)$ & $.64(.35,1.15)$ \\
\hline$\geq 4$ & $59(41.55 \%)$ & $83(58.45 \%)$ & 1 & \\
\hline \multicolumn{5}{|l|}{ Intestinal parasite } \\
\hline Yes & $23(51.11 \%)$ & $22(48.89 \%)$ & $1.90(.99,3.63)$ & $3.10(1.39,6.88) \times$ \\
\hline No & $75(35.55 \%)$ & $136(64.45 \%)$ & 1 & \\
\hline
\end{tabular}

*statistically significant at $p$-Value $<0.05$ 